\title{
CMOS Current-Mode Divider and Its Applications
}

\author{
Weihsing Liu, Shen-Iuan Liu, Senior Member, IEEE, and Shui-Ken Wei
}

\begin{abstract}
Compact, accurate and low-power analog CMOS circuits for current-mode division and pseudo-exponential function generation are presented, based on a new variable transresistance amplifier. Experimental results of the circuits fabricated in a 0.5- $\mu \mathrm{m}$ 2P2M n-well CMOS process show better than $0.3 \%$ total harmonic distortion. Measured power is less than $0.22 \mathrm{~mW}$ at $100-\mathrm{MHz}$ bandwidth and $\pm 1.5-\mathrm{V}$ supply voltages.
\end{abstract}

Index Terms-Divider, exponential function, transresistance.

\section{INTRODUCTION}

$\mathbf{T}$ HE ANALOG divider is an important building block in the design of analog signal processing integrated circuits, such as analog computation, fuzzy control, neural network, and analog-digital (A/D) converters and communication systems [1]-[6], etc. However, most of the analog dividers operate in the voltage mode [4], [7]-[9] and only few of them are designed to operate in the current mode [10], [11]. In the past decade, current-mode signal processing has received much attention for their potential advantages such as wide bandwidth, wider dynamic range, simple circuitry, and lower power consumption [12]. In this brief, a new CMOS current-mode divider is presented. Two different applications are presented to demonstrate the proposed current-mode divider. Experimental results of all the proposed circuits are given to verify the theoretical analysis.

\section{CIRCUIT DESCRIPTION}

Consider the circuit shown in Fig. 1 [13]. Assume that both M1 and M2 are biased in the triode region without body effect, their drain currents are given by [14]

$$
\begin{aligned}
& I_{1}=\frac{K_{n 1}}{2}\left(2\left(-V_{S S}-V_{\mathrm{Tn} 1}\right) V_{D S 1}-V_{D S 1}{ }^{2}\right) \\
& I_{2}=I_{\text {in }}+I_{4}=\frac{K_{n 2}}{2}\left(2\left(V_{G 2}-V_{S S}-V_{\mathrm{Tn} 2}\right) V_{D S 2}-V_{D S 2}{ }^{2}\right)
\end{aligned}
$$

where $V_{G 2}$ is a bias voltage, $K_{n 1,2}$ are the transconductance parameters and $V_{\operatorname{Tn} 1,2}$ are the threshold voltages of $\mathrm{M} 1$ and $\mathrm{M} 2$,

Manuscript received December 26, 2002; revised October 10, 2004. This work was supported in part by the National Science Council, Taiwan, R.O.C. under Grant NSC 90-2626-E-236-001. This paper was recommended by Associate Editor G. Cauwenberghs.

W. Liu is with the Department of Electrical Engineering and Graduate Institute of Electronics Engineering, National Taiwan University, Taipei 10617, Taiwan, R.O.C., and also with the Faculty of the Department of Electronic Engineering, Tung Nan Institute of Technology, Taipei 22202, Taiwan, R. O. C. (e-mail: whliu@mail.tnit.edu.tw).

S.-I. Liu is with the Department of Electrical Engineering and Graduate Institute of Electronics Engineering, National Taiwan University, Taipei 10617, Taiwan, R.O.C. (e-mail: 1si@cc.ee.ntu.edu.tw).

S.-K. Wei is with the Department of Electronic Engineering, Tung Nan Institute of Technology, Taipei 22202, Taiwan, R. O. C.

Digital Object Identifier 10.1109/TCSII.2004.842041

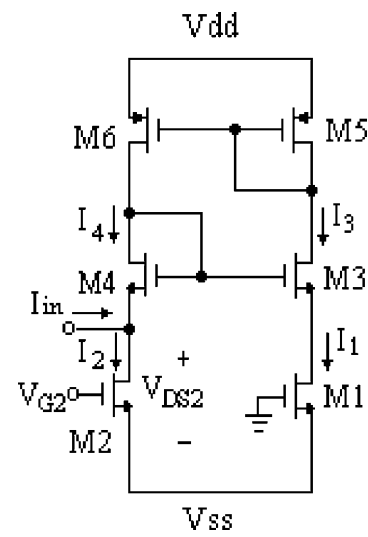

Fig. 1. Proposed voltage-controlled resistor.

respectively. The current mirrors M5 and M6 are used to generate the current $I_{3}$ and $I_{4}$, so that

$$
I_{3}=I_{4}=I_{1} \text {. }
$$

Assume that M3 and M4 are perfectly matched (i.e., $K_{n 3}=$ $K_{n 4}$ and $\left.V_{\mathrm{Tn} 3}=V_{\mathrm{Tn} 4}\right)$ and both of them are biased in saturation. According to the square-law characteristics of MOSFETs, the following are given [14]:

$$
V_{G S 3}=V_{G S 4}=\sqrt{\frac{2 I_{1}}{K_{n 3}}}+V_{\mathrm{Tn} 3} .
$$

Since $V_{G S 3}+V_{D S 1}=V_{G S 4}+V_{D S 2}$, it leads to

$$
V_{D S 1}=V_{D S 2} \text {. }
$$

Because source voltages of $\mathrm{M} 3$ and $\mathrm{M} 4$ are equal, i.e., $V_{\mathrm{SB} 3}=$ $V_{\mathrm{SB} 4}$, therefore the assumption that $V_{\mathrm{Tn} 3}=V_{\mathrm{Tn} 4}$ could be held. Substituting (1), (3), and (5) into (2) and assuming that M1 and M2 are perfectly matched (i.e., $K_{n 1}=K_{n 2}=K_{n}$ and $V_{\mathrm{Tn} 1}=$ $\left.V_{\mathrm{Tn} 2}=V_{\mathrm{Tn}}\right), I_{\mathrm{in}}$ is derived as

$$
I_{\text {in }}=K_{n} \cdot V_{G 2} \cdot V_{D S 2} .
$$

From (6), a voltage-controlled resistor may be given and its equivalent resistance is given as

$$
R_{\mathrm{eq}}=\frac{V_{D S 2}}{I_{\mathrm{in}}}=\frac{1}{K_{n} V_{G 2}} .
$$

According to (7), the equivalent resistance is reversely proportional to the bias voltage $V_{G 2}$.

Based on the proposed voltage-controlled resistor, the proposed current-mode divider is shown in Fig. 2. Assume that both M7 and M8 are biased in saturation, they can realize a current-to-voltage converter [15]. Assume that M7 and M8 are perfectly matched (i.e., $K_{p 7}=K_{p 8}=K_{p}$ and $V_{T p 7}=V_{T p 8}=$ $V_{T p}$ ) and both of them are embodied in individual wells to avoid the body effect. If the supply voltages $V_{\mathrm{DD}}=\left|V_{S S}\right|$, yields

$$
V_{G 2}=\frac{I_{8}-I_{7}}{2 K_{p}\left(V_{\mathrm{DD}}-\left|V_{T p}\right|\right)}=\frac{I_{D}}{2 K_{p}\left(V_{\mathrm{DD}}-\left|V_{T p}\right|\right)} .
$$




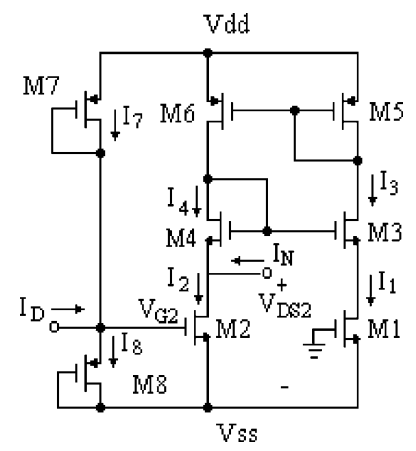

Fig. 2. Proposed current-mode divider.

Substituting (8) into (6) yields

$$
V_{D S 2}=\frac{2 K_{p}\left(V_{\mathrm{DD}}-\left|V_{T p}\right|\right)}{K_{n}} \cdot \frac{I_{N}}{I_{D}} .
$$

According to (9), a current-mode divider would be realized. However, the output voltage of the proposed current-mode divider is influenced by the variation of the supply voltage. To keep the proposed current-mode divider work properly, M1 and M2 should be biased in the triode region and other transistors should be in saturation. Since the current $I_{N}$ must flow into the drain of M2 therefore the gate voltage of M2 should be larger than the ground potential. According to (8), yields

$$
I_{D}>0 \text {. }
$$

Next, for M2 to operate in the triode region, the output range may be derived as

$$
V_{D S 2}<\frac{I_{D}}{2 K_{p}\left(V_{\mathrm{DD}}-\left|V_{T p}\right|\right)}-V_{\mathrm{Tn} 2}
$$

where $K_{p}$ is the transconductance of $\mathrm{M} 7$ and $\mathrm{M} 8$ and $V_{\mathrm{Tn} 2}$ is the threshold voltage of M2, respectively.

\section{APPLICATIONS}

\section{A. Pseudo-Exponential Function Generator}

A pseudo-exponential function may be written as [16]

$$
\exp (4 x) \cong\left(\frac{1+x}{1-x}\right)^{2} \quad ; \text { if }|\mathrm{x}| \ll 1 .
$$

The proposed pseudo-exponential function generator is shown in Fig. 3 [17]. Assume that M9 and M10 are perfectly matched (i.e., $K_{p 9}=K_{p 10}=K_{p}$ and $V_{T p 9}=V_{T p 10}=V_{T p}$ ) and both of M9 and M10 are biased in saturation. Based on the square-law characteristics of MOSFETs, the currents $I_{D}$ and $I_{N}$ are written as

$$
I_{D}=I_{9}=\frac{K_{p}}{2}\left(V_{\mathrm{DD}}-\left(V_{b}+V_{\text {in }}\right)-\left|V_{T p}\right|\right)^{2}
$$

and

$$
I_{N}=I_{10}=\frac{K_{p}}{2}\left(V_{\mathrm{DD}}-\left(V_{b}-V_{\mathrm{in}}\right)-\left|V_{T p}\right|\right)^{2}
$$

where $V_{b}$ is the bias voltage. Assume that $V_{b}<0$ and $V_{\mathrm{DD}}-$ $V_{b}>\left|V_{T p}\right|$. Substituting (13) and (14) into (9) yields

$$
V_{D S 2}=\frac{2 K_{p}\left(V_{\mathrm{DD}}-\left|V_{T p}\right|\right)}{K_{n}} \cdot\left(\frac{1+\frac{V_{\text {in }}}{V_{\mathrm{DD}}-V_{b}-\left|V_{T p}\right|}}{1-\frac{V_{\text {in }}}{V_{\mathrm{DD}}-V_{b}-\left|V_{T p}\right|}}\right)^{2} \text {. }
$$

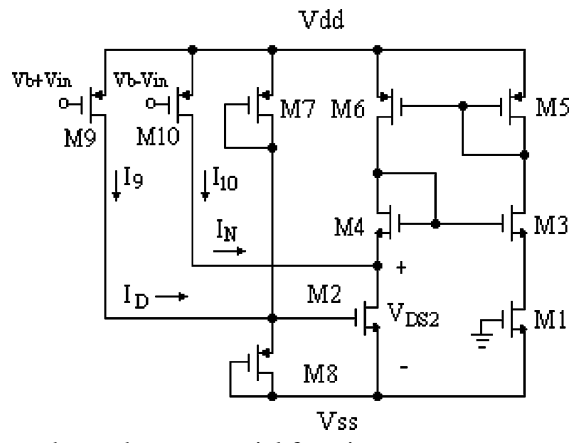

Fig. 3. Proposed pseudo-exponential function generator.

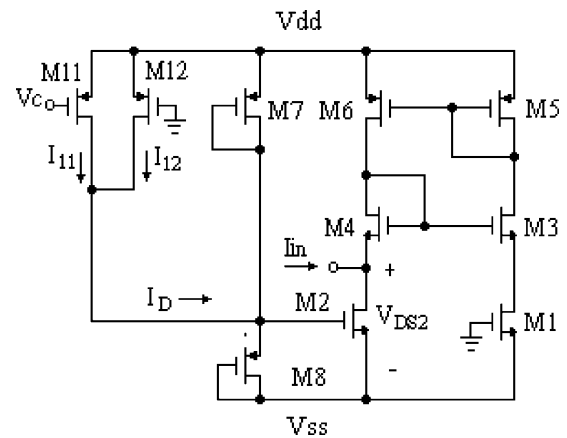

Fig. 4. Proposed variable transresistance amplifier.

Comparing (15) with (12), results in

$$
V_{D S 2}=b \cdot\left(\frac{1+a V_{\text {in }}}{1-a V_{\text {in }}}\right)^{2} \cong b \cdot \exp \left(4 a V_{\text {in }}\right) \quad \text { if }\left|a V_{\text {in }}\right| \ll 1
$$

where $a=1 /\left(V_{\mathrm{DD}}-V_{b}-\left|V_{T p}\right|\right)$ and $b=2\left(K_{p} / K_{n}\right)\left(V_{\mathrm{DD}}-\right.$ $\left.\left|V_{T p}\right|\right)$. According to (16), a pseudo-exponential function generator may be realized.

\section{B. Variable Transresistance Amplifier}

The proposed variable transresistance amplifier is shown in Fig. 4. M11 and M12 realized an exponential function generator [18]. Assume that M11 and M12 are biased in saturation and the transconductance parameters and the threshold voltages of M11 and M12 are equal (i.e., $K_{p 11}=K_{p 12}=K_{p}$ and $V_{T p 11}=$ $V_{T p 12}=V_{T p}$ ). According to the square-law characteristics of the MOSFETs, the currents $I_{11}$ and $I_{12}$ are given as [14]

$$
\begin{aligned}
& I_{11}=\frac{K_{p}}{2}\left(V_{\mathrm{DD}}-V_{c}-\left|V_{T p}\right|\right)^{2} \\
& I_{12}=\frac{K_{p}}{2}\left(V_{\mathrm{DD}}-\left|V_{T p}\right|\right)^{2} .
\end{aligned}
$$

According to the assumptions $|(a / b) x| \ll 1$ and $b^{2}+(b+$ $a x)^{2} \cong \exp ((a / b) x)$ [18], the current $I_{D}$ is given as

$$
\begin{aligned}
I_{D} & =I_{11}+I_{12} \\
& =K_{p}\left(V_{\mathrm{DD}}-\left|V_{T p}\right|\right)^{2}\left(\frac{V_{c}^{2}}{2\left(V_{\mathrm{DD}}-\left|V_{T p}\right|\right)^{2}}-\frac{V_{c}}{\left(V_{\mathrm{DD}}-\left|V_{T p}\right|\right)}+1\right) \\
& \cong I_{b 1} \cdot \exp \left(-a_{1} V_{c}\right)
\end{aligned}
$$

where $I_{b 1}=K_{p}\left(V_{\mathrm{DD}}-\left|V_{T p}\right|\right)^{2}, a_{1}=1 /\left(V_{\mathrm{DD}}-\left|V_{T p}\right|\right)$ and $V_{c}$ is a control voltage. Substituting (19) into (8) and let the current $I_{N}$ be the input current $I_{\text {in }}$ yields

$$
V_{D S 2} \cong \frac{2}{K_{n}\left(V_{\mathrm{DD}}-\left|V_{T p}\right|\right)} I_{\text {in }} \cdot \exp \left(a_{1} V_{c}\right) .
$$

Therefore, a variable transresistance amplifier may be realized. 


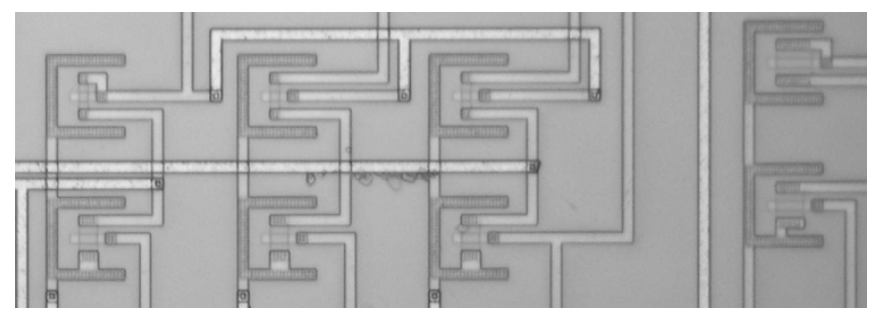

Fig. 5. Die photograph of the proposed current-mode divider.

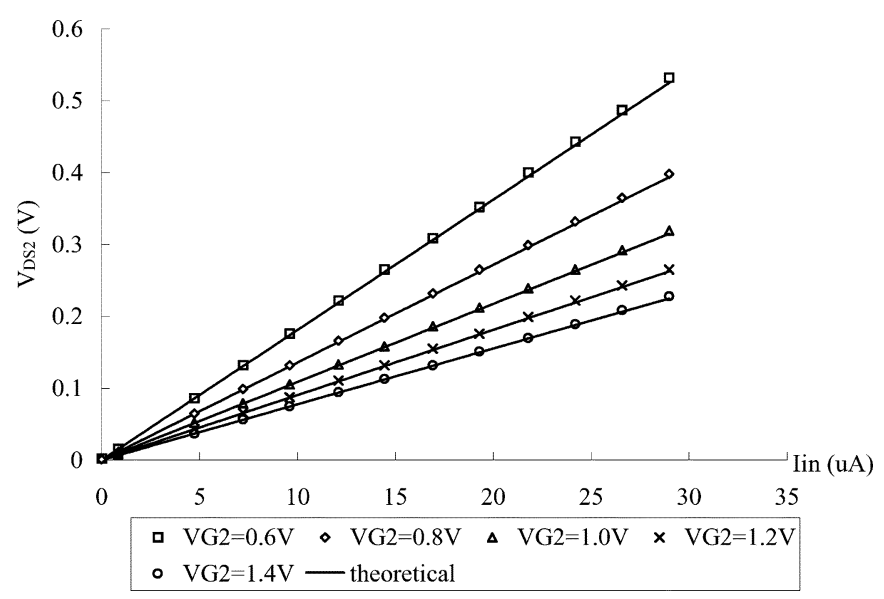

Fig. 6. Experimental results of the proposed voltage-controlled resistor.

\section{EXPERIMENTAL RESULTS}

All the proposed circuits have been fabricated in a $0.5-\mu \mathrm{m}$ $2 \mathrm{P} 2 \mathrm{M}$ N-well CMOS process. The die photograph of the current-mode divider is shown in Fig. 5. The threshold voltage for the NMOS is $0.78 \mathrm{~V}$ and that for the PMOS is $-1.1 \mathrm{~V}$ in our process and the transconductance parameters in our process are $K_{n}=2.02 K_{p}=0.186 \mathrm{~mA} / \mathrm{V}^{2}$. All the experiments were performed with supply voltages $V_{\mathrm{DD}}=\left|V_{\mathrm{ss}}\right|=1.5 \mathrm{~V}$.

Fig. 6 shows the experimental results of the proposed voltagecontrolled resistor which were performed with the bias voltages $V_{G 2}=0.6,0.8,1.0,1.2$, and $1.4 \mathrm{~V}$, respectively. As the input current $I_{\text {in }}$ varies from $0 \sim 30 \mu \mathrm{A}$, the measured equivalent resistors are $18.44 \mathrm{k} \Omega$ (at $V_{G 2}=0.6 \mathrm{~V}$ ), $13.76 \mathrm{k} \Omega$ (at $V_{G 2}=$ $0.8 \mathrm{~V}), 10.87 \mathrm{k} \Omega$ (at $\left.V_{G 2}=1.0 \mathrm{~V}\right), 9.22 \mathrm{k} \Omega$ (at $V_{G 2}=1.2 \mathrm{~V}$ ) and $7.85 \mathrm{k} \Omega$ (at $V_{G 2}=1.4 \mathrm{~V}$ ), respectively, which is consistent with the theoretical analysis calculated by (7).

The experimental results of the proposed current-mode divider are shown in Fig. 7(a) and (b) which have been performed with $I_{D}=, 40,50,70$, and $80 \mu \mathrm{A}$ while $I_{N}$ varies from $0 \sim$ $30 \mu \mathrm{A}$ and $I_{N}=10,15,20,25$, and $30 \mu \mathrm{A}$ while $I_{D}$ varies from $1 \sim 70 \mu \mathrm{A}$, respectively. The measured output offset voltage is less than $1.5 \mathrm{mV}$ under all situations and the measured maximum linear error is less than $0.85 \%$. The measured total harmonic distortion (THD) at $100 \mathrm{kHz}$ of the output voltage for $I_{D}=30 \mu \mathrm{A}$ and the amplitude of the current $I_{N}$ is 0.1 , 0.5 , and $1 \mu \mathrm{A}$ are $0.03 \%, 0.122 \%$, and $0.243 \%$, respectively. Also, the measured power consumption is less than $0.22 \mathrm{~mW}$ (at $\left.I_{D}=I_{N}=30 \mu \mathrm{A}\right)$. For the proposed current-mode divider to work properly, the current $I_{D}$ must be limited by (10) and (11). As the current $I_{D}$ was increased to $86 \mu \mathrm{A}$, the corresponding output deviated from its theoretical value [calculated by (9)] by

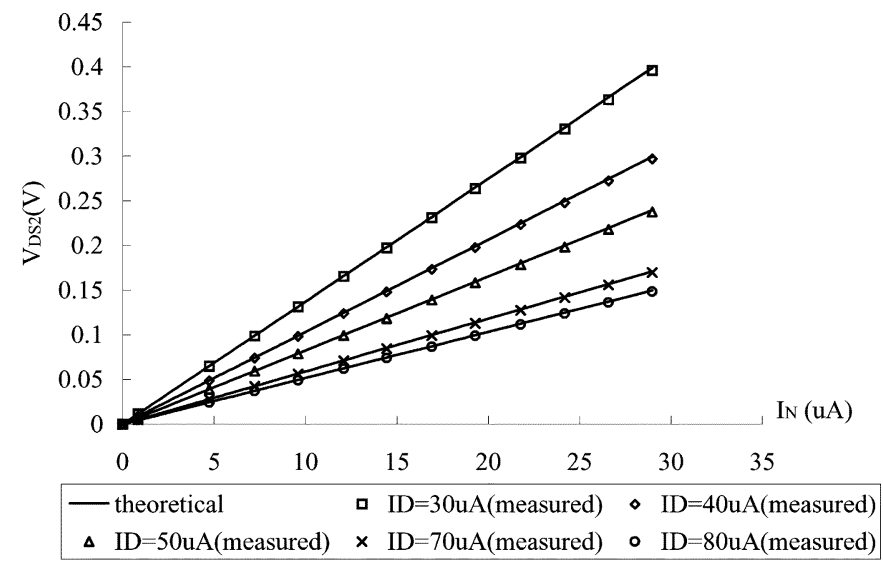

(a)

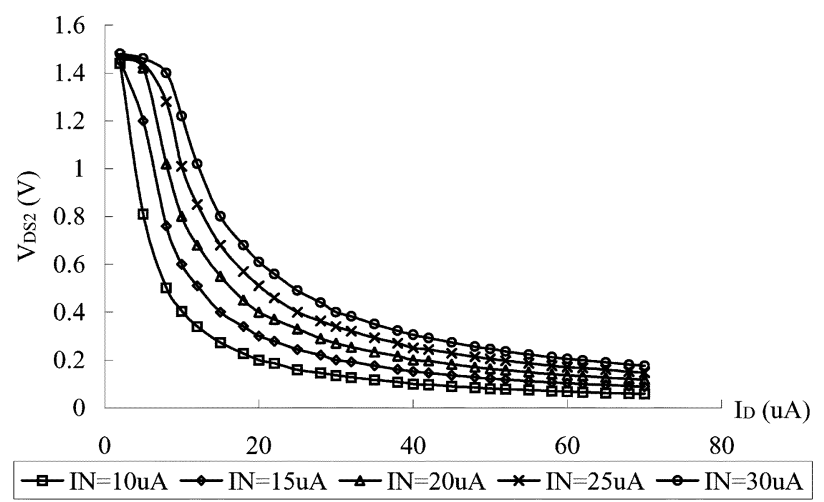

(b)

Fig. 7. Experimental results of the current-mode divider (a) $\mathrm{V}_{D S 2}$ vs. $I_{N}$ (b) $V_{D S 2}$ vs. $I_{D}$.

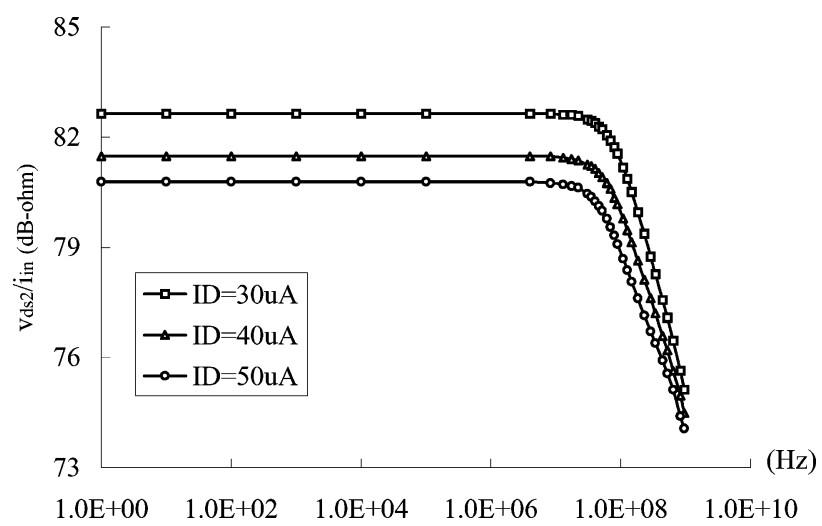

Fig. 8. Frequency response of the proposed current-mode divider.

$2.78 \%\left(\right.$ at $\left.I_{N}=30 \mu \mathrm{A}\right)$. The experimental results are consistent with the theoretical analysis calculated by (9). For measuring the power-supply rejection ratio (PSRR), assume that a ripple was generated along with the supply voltage $V_{\mathrm{DD}}$. If the magnitude of the ripple is $10 \%$ of the $V_{\mathrm{DD}}$ and the frequency is $100 \mathrm{kHz}$, the measured PSRR is about $26.67 \mathrm{~dB}$. The frequency response of Fig. 2 is shown in Fig. 8 which was performed with the current $I_{D}=30,40$, and $50 \mu \mathrm{A}$ while the current $I_{N}=20 \mu \mathrm{A}$ and the small-signal current was set to $1 \%$ of the current $I_{N}$. The corresponding -3-dB bandwidth are 231, 191, and $179 \mathrm{MHz}$, respectively. Also, the measured input referred noise (at $100 \mathrm{kHz}$ ) is $1.49 \times 10^{-8} \mathrm{~A} / \sqrt{ } \mathrm{Hz}$. 


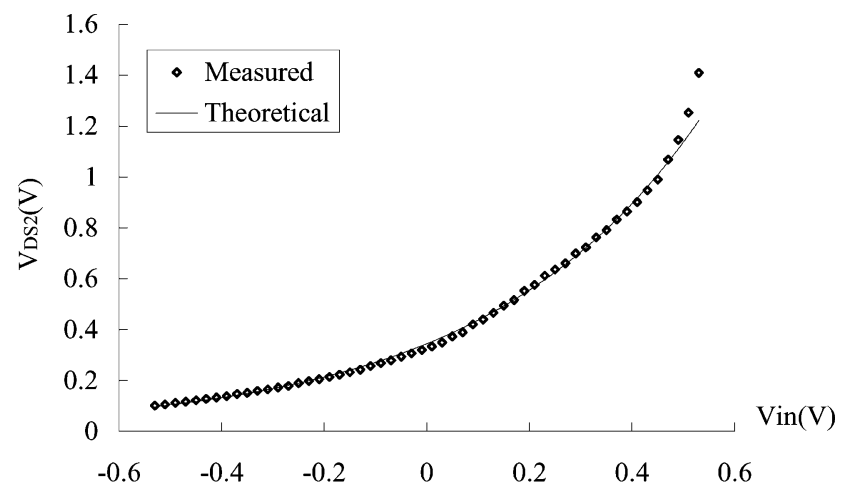

(a)

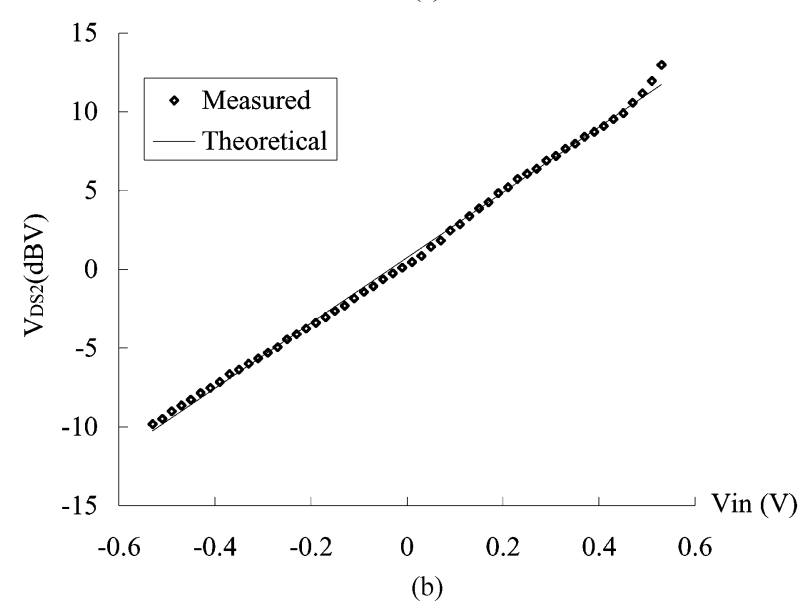

Fig. 9. Experimental results of the proposed pseudo-exponential function generator. (a) Linear scale. (b) Semi-logarithmic (dB) scale.

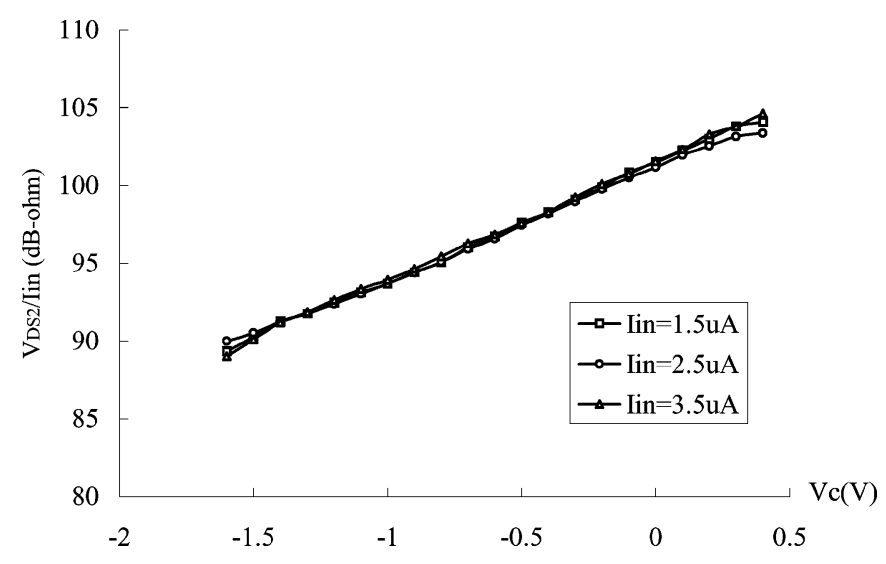

Fig. 10. Experimental results of the proposed variable transresistance amplifier.

The experimental results of the proposed pseudo-exponential function generator are shown in Fig. 9(a) and (b), respectively. With the bias voltage $V_{b}=-0.4 \mathrm{~V}$, as $V_{\text {in }}$ varies from -0.5 to $0.5 \mathrm{~V}$, the output operating range could be more than $20 \mathrm{~dB}$ while the linearity error is less than $\pm 0.5 \mathrm{~dB}$. The experimental results confirm the theoretical analysis calculated by (16).
Fig. 10 shows the experimental results of the proposed variable transresistance amplifier. The experiments were performed with the input currents $I_{\text {in }}=1.5,2.5$, and $3.5 \mu \mathrm{A}$, respectively. As the control voltage $V_{c}$ varies from -1.6 to $0.4 \mathrm{~V}$, the transresistance ranges from 90 to $102 \mathrm{~dB} \Omega$ while the linearity error is less than $\pm 1 \mathrm{~dB}$. The experimental results verify the theoretical analysis calculated by (20). As a comparison, two different samples were tested using the same procedure, with very similar results.

\section{CONCLUSION}

In this brief, a new CMOS current-mode divider is developed. Experimental results have been given to confirm the validity of the theoretical analysis. The proposed current-mode divider can be used to realize a voltage-controlled resistor, a pseudo-exponential function generator and a variable transresistance amplifier.

\section{REFERENCES}

[1] C. Mead and M. Ismail, Analog VLSI Implementation of Neural Systems. Norwell, MA: Kluwer, 1989.

[2] Nonlinear Circuit Handbook, D. H. Ssheingold, Ed., Analog Devices, Norwood, MA, 1974.

[3] F. J. Pelayo, I. Rojas, J. Ortega, and A. Priteo, "Current-mode analogue defuzzifier," Electron. Lett., vol. 29, no. 9, pp. 743-744, 1993.

[4] P. Deval, G. Wegmann, and J. Robert, "CMOS pipelined A/D convertor using current divider," Electron. Lett., vol. 25, no. 20, pp. 1341-1343, 1989.

[5] U. Singh and M. Green, "Dynamics of high-frequency CMOS dividers," in Proc. IEEE Int. Symp. Circuits Systems, vol. 5, May 2002, pp. $421-424$.

[6] - ,New structures for very high-frequency CMOS clock dividers," in Proc. IEEE Int. Symp. Circuits Systems, vol. 4, May 2002, pp. 622-625.

[7] D. Ghosh and P. Patranabis, "A simple analog divider having independent control of sensitivity and design conditions," IEEE Trans. Instrum. Meas., vol. 39, no. 3, pp. 522-526, Jun. 1990.

[8] S. I. Liu and C. C. Chang, "CMOS analog divider and four-quadrant multiplierusing pool circuits," IEEE J. Solid-State Circuits, vol. 30, no. 9, pp. 1025-1029, Sep. 1995.

[9] J. G. Graeme, Operational Amplifier Design and Application. New York: McGraw-Hill, 1971.

[10] H. Wasaki, Y. Horio, and S. Nakamura, "Current multiplier/divider circuit," Electron. Lett., vol. 27, no. 6, pp. 504-506, 1991.

[11] C. H. J. Mensink and B. Nauta, "CMOS tunable linear current divider," Electron. Lett., vol. 32, no. 10, pp. 889-890, 1996.

[12] B. Wilson, "Recent developments in current conveyor and current-mode circuits," Proc. Inst. Elect. Eng. G, vol. 137, no. 2, pp. 63-77, 1990.

[13] W. Liu and S. I. Liu, "CMOS tunable 1/x circuit and its applications," IEICE Trans. Fund., vol. E-86A, pp. 1896-1899, Jul. 2003.

[14] N. R. Malik, Electronic Circuits. Englewoood Cliffs, NJ: PrenticeHall, 1995.

[15] K. Bult and H. Wallinga, "A class of analog CMOS circuits based on the square law characteristics of an MOS transistor in saturation," IEEE J. Solid-State Circuits, vol. SC-22, pp. 357-365, Jun. 1987.

[16] A. Motamed, C. Hwang, and M. Ismail, "CMOS exponential current-tovoltage converter," Electron. Lett., vol. 33, no. 12, pp. 998-1000, 1997.

[17] W. Liu and S. I. Liu, "CMOS exponential function generator," Electron. Lett., vol. 39, pp. 1-2, Jan. 2003.

[18] C. C. Chang and S. I. Liu, "Pseudo-exponential function using MOSFETs in saturation," IEEE Trans. Circuits Systems II, Analog Digit. Signal Process., vol. 47, pp. 1318-1321, Nov. 2000. 